\title{
3-D STUDY OF VESSELS IN PERIPHERAL PLACENTAL VILLI
}

\author{
MARIE JIRKOVSKÁ ${ }^{1}$, LUCIE KUBÍNOVÁ ${ }^{2}$, JiŘí JANÁČEK ${ }^{2}$ AND JOSEF KALÁB ${ }^{1,2}$ \\ ${ }^{1}$ Institute of Histology and Embryology, First Faculty of Medicine, Charles University in Prague, Albertov 4, \\ Prague 2, CZ-12800, Czech Republic; ${ }^{2}$ Department of Biomathematics, Institute of Physiology, Academy of \\ Sciences of the Czech Republic, Vídeňská 1083, Prague 4, CZ-14220, Czech Republic \\ e-mail: mjirk@lf1.cuni.cz; kubinova@biomed.cas.cz; janacek@biomed.cas.cz \\ (Accepted October 10, 2007)
}

\begin{abstract}
The microvascular bed of the human term placenta is located in the mature intermediate and terminal villi. Despite repeated studies, a commonly accepted model of its spatial organization is not yet available. In the present study, the 3-D arrangement of vessels in mature intermediate and terminal placental villi was examined using the combination of confocal microscopy and image processing. Fixed and paraffin embedded placental samples were cut into $120 \mu \mathrm{m}$ thick sections. Serial optical sections were captured by a confocal microscope and used as an initial data for 3-D reconstruction. The reconstructed segments of the villous tree show parallel vessels of mature intermediate villi giving rise to the capillaries of terminal villi. The signs of longitudinal and sprouting capillary growth as well as growth of new villi were observed. The presented method allowed us to visualize large parts of peripheral placental villi, which is useful for elucidating the arrangement of their vascular bed.
\end{abstract}

Keywords: 3-D image processing, 3-D reconstruction, angiogenesis, capillaries, confocal microscopy, placental villi.

\section{INTRODUCTION}

The fetoplacental vascular bed serves as a means of transport of nutrients and metabolites from placenta to fetus and vice versa. In the human villous placenta, it is located inside tree-like placental villi. As the villi ramify, they become thinner and the vessels located inside them change their diameter and composition of the wall. In the placenta at term, peripheral parts of the villous tree consist of mature intermediate and terminal villi encompassing the microvascular placental bed. The mature intermediate villi contain supplying arterioles, thin capillaries and venules. The vast majority of placental capillary bed is situated in terminal villi and arises from these vessels. Capillaries and villous trophoblast constitute placental barrier which not only separates the maternal and fetal blood but also fulfills a transport role. As fetal nutrients requirements increase and the surface area of capillary wall is decisive for the amount of transported substances, placental capillaries grow up to the end of pregnancy.

The structure and function of microvessels in normal and pathological placenta is the subject of interest to many researchers. Nevertheless, the spatial arrangement of placental microvascular bed has been dealt with only in a few publications in the last fifty years. Two authors injected a dye solution into placental vessels and observed its distribution using a light microscope (Crawford, 1956; Boe, 1969). The models based on these observations are contradictory. While the former described villous capillary as a long nonbranched garland running through grape-like arranged terminal villi, the latter shows a complicated, richly ramified capillary bed of terminal villi connected with dense paravascular capillaries of mature intermediate villi.

Another model based on corrosive casts (Arts, 1961) is represented by sparse paravascular capillaries in mature intermediate villi and longitudinally oriented capillaries connected with numerous short connections in terminal villi.

The authors of so far latest studies (Kaufmann et al., 1985; Leiser et al., 1985) applied the method of physical three-dimensional (3-D) reconstruction based on serial histological sections. In terminal villi, their model contains U-shaped capillary loops interconnected here and there with short connections. The loops are running out from the arterioles or capillaries of the mature intermediate villus.

In summary, although the methods used for such studies were obviously up to date, the results and 
interpretations provide a rather incoherent picture. Due to their inherent limitations, the methods used so far did not allow to study spatial organization of villous vessels, neither in relation to other tissues, nor in a larger amount of samples. Therefore, a commonly accepted model of the spatial organization of vessels in peripheral parts of villous tree, namely in mature intermediate and terminal villi, is still lacking.

In this study, we aim to contribute to the elucidation of the arrangement of the placental microvascular bed by 3-D renderings of villous vessels, using confocal microscopy and tools of computer-based visualization.

\section{MATERIALS AND METHODS}

\section{TISSUE SECTIONS}

Samples of human term placentas were fixed in a fixative (8 ml glutaraldehyde 25\%, $20 \mathrm{ml}$ formaldehyde $36 \%, 2.62$ g dried sodium phosphate, $170 \mathrm{ml}$ water and $0.5 \%$ eosin, $\mathrm{pH}$ adjusted at 7.2 ) for $24 \mathrm{hrs}$, and embedded in paraffin. Sections, about $120 \mu \mathrm{m}$ thick, were cut using a microtome HM340 E (MICROM Laborgeräte, Walldorf, Germany), deparaffinized and mounted.

\section{CONFOCAL MICROSCOPY}

Series of thin optical sections ( $1 \mu \mathrm{m}$ apart) of villous segments lying completely inside the thick sections were recorded by a BioRad MRC 600 confocal laser scanning microscope using a planapochromat oil immersion objective with 40x magnification (N.A. $=1.3$ ) and used as the initial data for 3-D demonstration of spatial arrangement of capillaries inside the villi. If the analyzed segment of the villous tree was too large, two overlapping series of optical sections were captured and subsequently composed using our GlueMRC programme (Karen et al., 2003).

\section{3-D RECONSTRUCTION}

The contours of villi and capillaries were drawn by hand using the Ellipse Colon module (ViDiTo, SLOVAKIA) because the low contrast of the images did not allow automated segmentation. The coordinates of the capillaries were converted into 3-D binary images by drawing the contours as filled polygons. To smooth the irregular surfaces of the objects composed from the hand drawn contours, the image was resampled to grey image with lower resolution and treated by a 3-D Gaussian filter. The triangulated surface of the constant grey value was detected by standard "marching cube" algorithm (Lorensen and Cline, 1987). Partial models of villous surface and capillaries were combined and the images of resulting 3-D scenes were rendered. The data was processed and rendered using visual programming environment IRIS Explorer (NAG, UK) supplied with special modules for 3-D image processing (Janáček and Kubínová, 1998).

\section{RESULTS}

The presented methods allowed us to visualize numerous segments of placental villous trees. As documented here, it was possible not only to analyze the 3-D arrangement of the villous vascular bed but also to observe manifestations of the capillary and villous growth.

The parallel vessels of mature intermediate villus markedly differ in their diameter and some of them are branched. The elongation of these vessels gives rise to a U-shaped capillary which is accompanied by the formation of a new terminal villus (Fig. 1).

Further branching of a simple capillary loop results in forming a more complicated capillary bed, consisting of longitudinally oriented branches interconnected by short capillary segments (Fig. 2). Markedly dilated capillary parts are located predominantly at the tips of villi.

Besides the longitudinal growth, branching of already existing capillary segments represents another way of placental angiogenesis. It displays itself by shorter or longer blindly ending sprouts inducing bulges of trophoblast observable on the villous surface (Fig. 1 ). The longer sprouts turn and finally unite with another capillary contributing both to the enlargement of available capillary wall area and to the elongation of terminal villi (Fig. 3). 


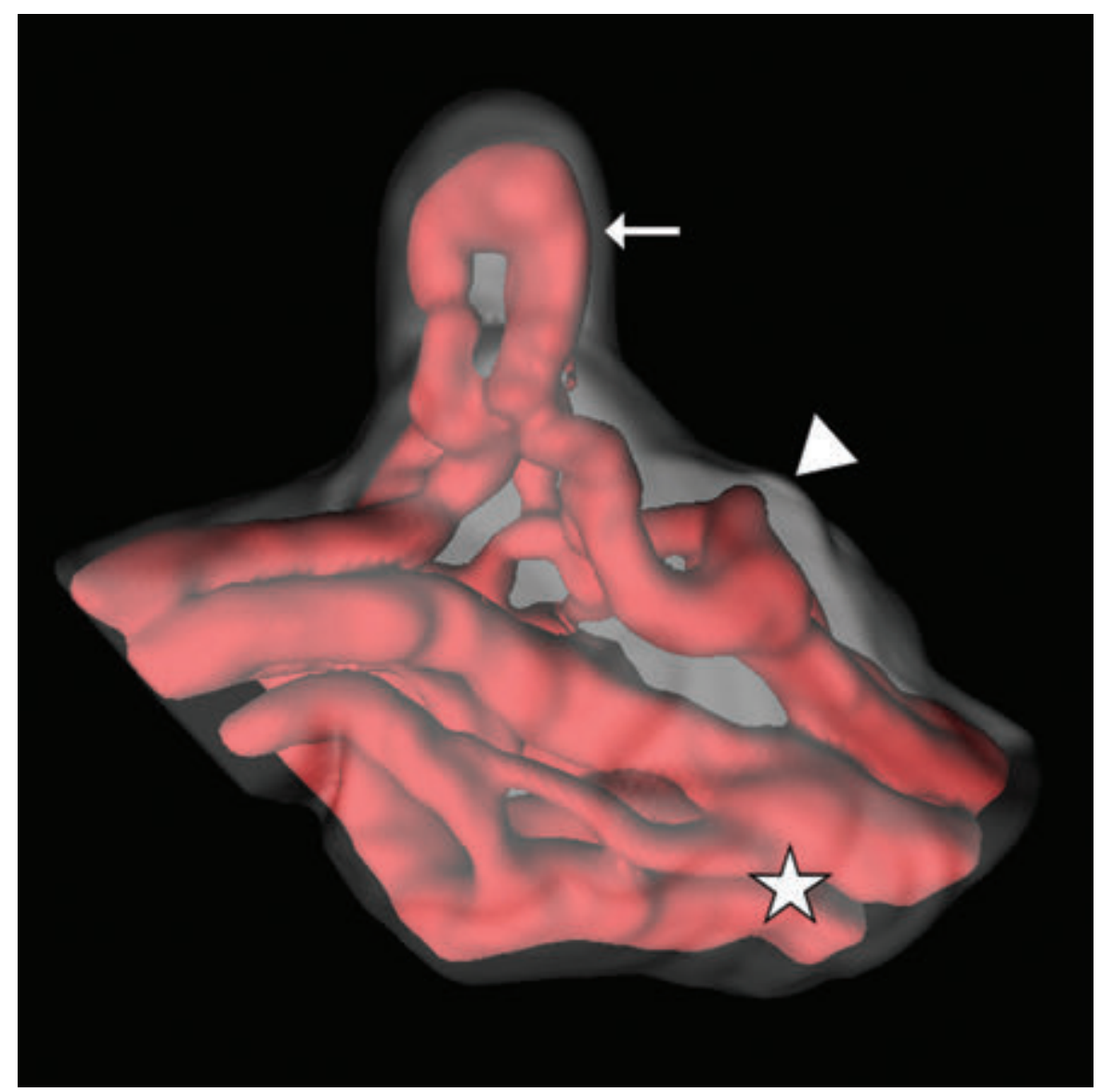

Fig. 1. Surface rendering of a segment of the peripheral placental villus. Asterisk: parallel arrangement of vessels in the mature intermediate villus; arrow: simple capillary loop in a terminal villus; arrowhead: bulging area of trophoblast containing capillary sprout.

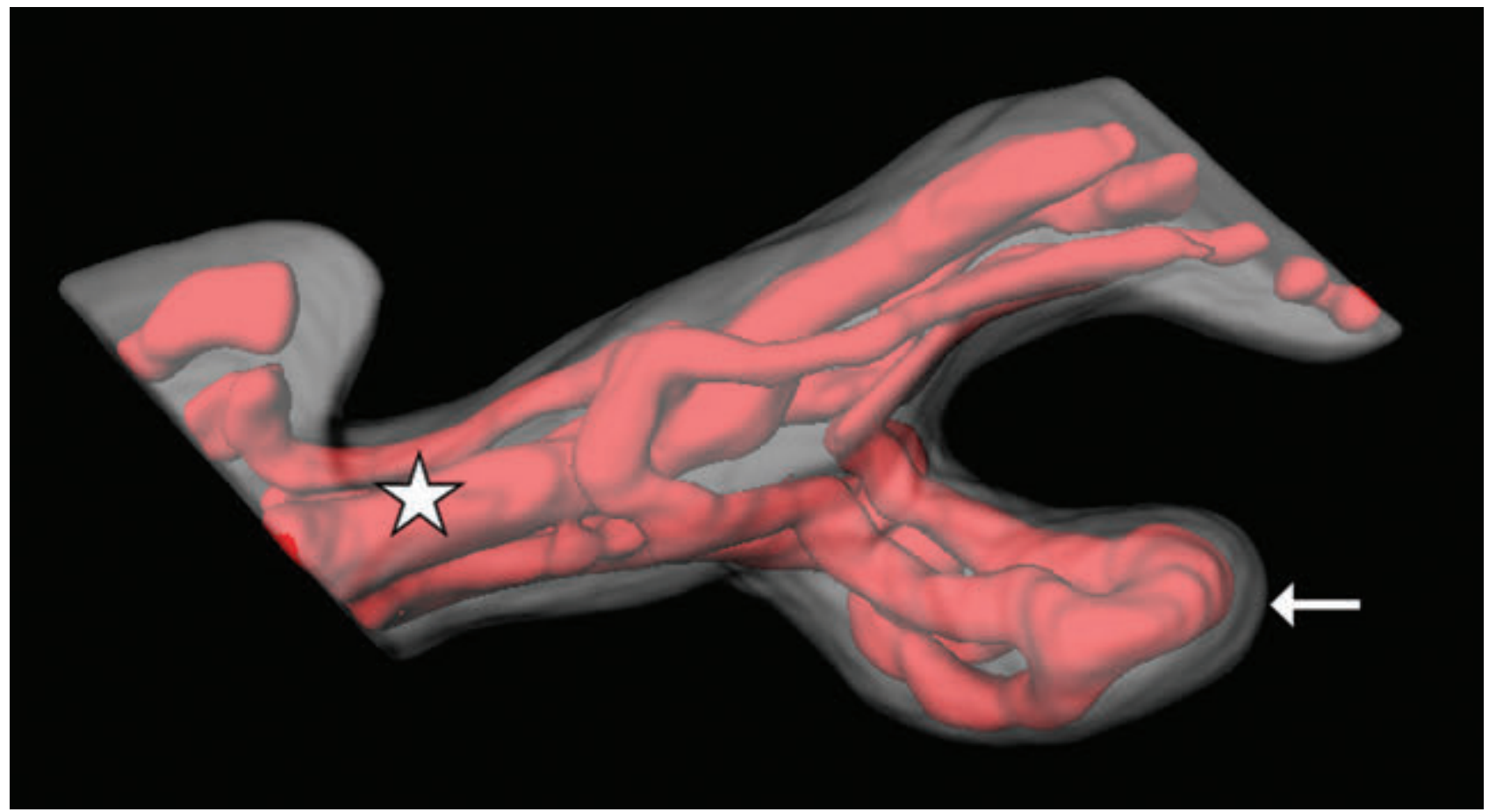

Fig. 2. Terminal villus displaying a more complicated capillary bed (arrow) connected to the capillaries of the mature intermediate villus (asterisk). The capillary in the tip of villus is markedly dilated. 


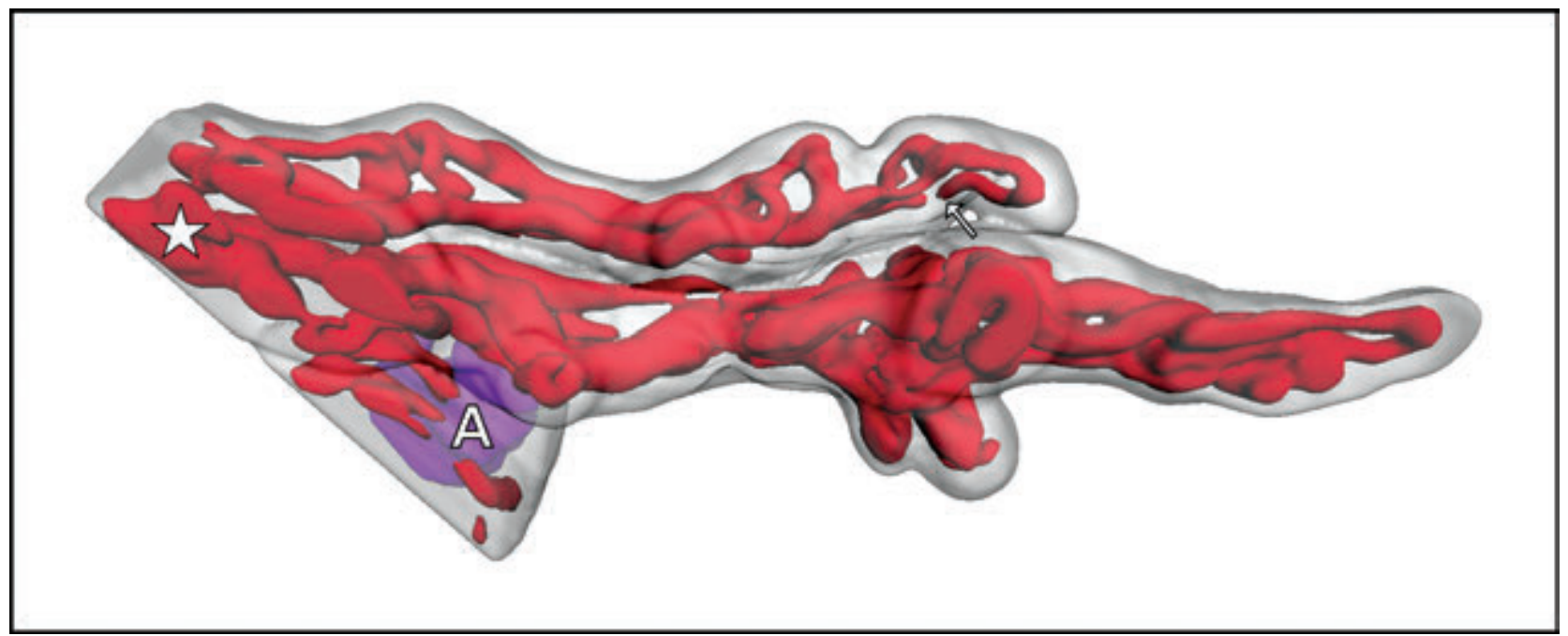

Fig. 3. Arteriole (A) and capillaries of the mature terminal villus (asterisk) give rise to the vascular bed of two tightly packed terminal villi. In the upper one, a long U-shaped sprout forms a new capillary loop (arrow). A stack of confocal optical sections composed from two overlapping stacks was used for the 3-D reconstruction.

\section{DISCUSSION}

Unlike many organs, where the microvessels resemble a spatial network, the most important part of the placental microvascular bed is located in well defined villi. As the fetal growth is followed by the growth of all placental constituents, placenta belongs to those rare organs, where intense physiological angiogenesis takes place. It was demonstrated that the continuity of growing villous vascular network is ensured by both the longitudinal and branching capillary growth. The elongation of capillary segments is attended by development of new terminal branches of the villous tree. Further longitudinal growth and branching of capillaries inside a newly formed villus as well as the formation of new villi enlarges the total areas of capillary wall and trophoblast available for materno-fetal transport. As shown previously in terminal villi, the angiogenic activity is adaptable and reacts on maternal disorders during pregnancy (Jirkovská et al., 2002).

The presented method allowed us to visualize large parts of peripheral placental villi, and thus proved to be a suitable tool for elucidation of the arrangement of the villous capillary bed.

\section{ACKNOWLEDGEMENTS}

This work was supported by the Grant Agency of the Czech Republic, Project No. 304/05/0153, and by the Academy of Sciences of the Czech Republic (AV0Z 50110509).

\section{REFERENCES}

Arts NFT (1961). Investigations on the vascular system of the placenta. I. General introduction and the fetal vascular system. Am J Obst Gynec 82:147-58.

Boe F (1969). Studies on the human placenta. III. Vascularization of the young fetal placenta. A. Vascularization of the chorionic villus. Acta Obstet Gynecol Scand 48: 159-66.

Crawford JM (1956). The foetal placenta circulation. IV. The anatomy of the villus and its capillary structure. $\mathrm{J}$ Obstet Gynaecol 63:548-52.

Janáček J, Kubínová L (1998). 3D reconstruction of surfaces captured by confocal microscopy. Acta Stereol 17:25964.

Jirkovská M, Kubínová L, Janáček J, Moravcová M, Krejčí V, Karen P (2002). Topological properties and spatial organization of villous capillaries in normal and diabetic placentas. J Vasc Res 39/3:268-78.

Kaufmann P, Bruns U, Leiser R, Luckhardt M, Winterhager E (1985). The fetal vascularisation of term human placenta villi. II. Intermediate and terminal villi. Anat Embryol 173:203-14.

Karen P, Jirkovská M, Tomori Z, Demjénová E, Janáček J, Kubínová L (2003). Three-dimensional computer reconstruction of large tissue volumes based on composing series of high- resolution confocal images by GlueMRC and LinkMRC software. Microsc Res Tech 62:415-22.

Leiser R, Luckhardt M, Kaufmann P, Winterhager E, Bruns U (1985). The fetal vascularisation of term human placenta villi. I. Peripheral stem villi. Anat Embryol 173: 71-80.

Lorensen WE, Cline HE (1987). Marching cubes: a high resolution 3D surface construction algorithm. Computer Graphics 21:163-169. 